\title{
2D-Nanomaterials for Controlling Friction and Wear at Interfaces
}

\author{
Jessica C. Spear, Bradley W. Ewers, and James D. Batteas* \\ Department of Chemistry, Texas A\&M University \\ PO Box 30012, College Station, TX 77842-3012
}

\author{
*Corresponding author: Email: batteas@chem.tamu.edu \\ Phone: 1-979-458-2958 \\ Fax: 1-979-845-3385
}

\begin{abstract}
This review focuses on recent developments in the use of 2D nanomaterials for controlling the frictional properties of surfaces and interfaces. While materials such as $\mathrm{MoS}_{2}$ and graphite have been investigated for some time, a host of other layered nanomaterials have emerged as alternatives for friction modification. These advanced lubrication schemes provide an opportunity to address growing needs in energy and materials efficiency and device compatibility, offering improved boundary and solid lubrication of contacting and sliding interfaces. Here, we describe both computational and experimental investigations of the mechanisms and implementations of 2D nanomaterials in the lubrication of interfaces.
\end{abstract}

Keywords: tribology, graphene, two dimensional materials, solid lubrication, boundary lubrication 


\section{Introduction}

Control of friction and wear are widely recognized problems that impact a broad range of materials applications from gears and engine components, to medical implants for joint replacement, to micro- and nano-scaled machine technologies, such as micro - and nano - electromechanical systems (MEMS, NEMS) devices. The nearly ubiquitous impact of friction and wear in numerous sectors of the economy (Figure 1) persistently motivates research into lubrication and surface coating schemes that more effectively address these challenges. At its core, this research is poised to proffer significantly improved efficiencies in the generation and usage of energy, one of society's most fundamental needs. Layered materials like graphite, $\mathrm{MoS}_{2}$, and hexagonal boron nitride (h-BN) have long been recognized as effective surface lubricants [1-3]. Offering the advantages of being solids, these lubricious films can be applied to open systems and can also be applied in other circumstances in which liquid lubrication is not appropriate. In all cases they offer interlayer sliding interfaces with phenomenally low interfacial shear strengths facilitated by characteristically weak interlayer interactions within the materials $[4,5]$.

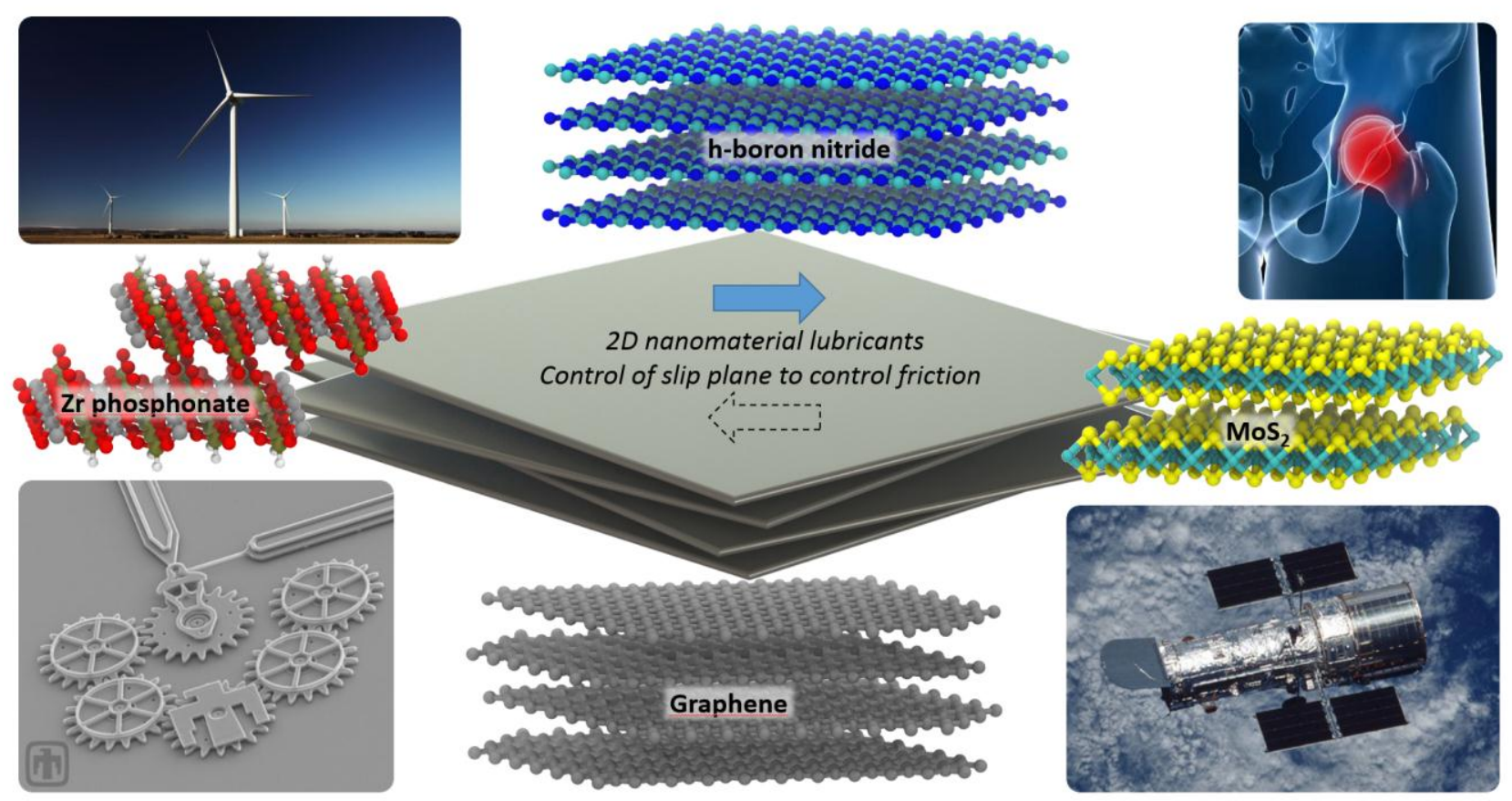

Figure 1. Layered materials like graphite, molybdenum disulfide, hexagonal boron nitride, and zirconium phosphates offer solutions to lubrication challenges in a variety of economically and technologically critical sectors of society. Pictures courtesy of NIH Senior Health, Sandia National Laboratories, SUMMiT ${ }^{\mathrm{TM}}$ Technologies, www.mems.sandia.gov, and NASA and the Space Telescope Science Institute. 
Recently, the ability to isolate single atomic layers of these materials has promoted an interesting new area of research into their frictional properties. Since its discovery in 2004 [6], graphene in particular has drawn considerable attention. Made up of a simple honeycomb carbon lattice, it has been found to exhibit many unique electronic and mechanical properties including ballistic electron transport [7], high thermal conductivity [8-11], large in-plane elastic modulus [12] and a low coefficient of friction [13-15]. These desirable properties make graphene of considerable interest for potential use in many applications including composite materials [16], energy transfer [17] and storage [18], electronic [19] and mechanical devices [20], and solid-state lubrication [13-15, 21]. Although mechanical exfoliation produces the most pristine, defect free graphene (edge sites not withstanding), it is not feasible for bulk production and large scale applications. Chemical vapor deposition (CVD) has been utilized as an alternative means to fabricate large area graphene by thermal decomposition of silicon carbide ( $\mathrm{SiC}$ ) and by its catalyzed growth on various metal surfaces [22]. Interestingly, there is also evidence that graphitic carbon can be generated in situ within sliding contacts which could provide a mechanism by which such surface coatings could be formed and repaired [23]. When considering utilizing graphene in real devices however, it must be recognized that its electronic and mechanical properties strongly depend on the interfacial interaction with the underlying substrate $[24,25]$. Due to its high out-of-plane flexibility, the morphology of graphene is dictated by the substrate geometry, which influences the measured properties through changes in electronic structure, topological defects, and chemical doping effects [26-31], and the frictional properties of graphene have been reported to have a unique dependence on the substrate surface roughness and interfacial adhesion [32-34].

With the isolation of graphene, the study of friction of single atomic layers could be pursued, offering potentially the thinnest feasible surface coating one could use as a lubricant. Akin to graphene, a host of other layered inorganic materials (e.g. h-BN, $\mathrm{MoS}_{2}, \mathrm{Y}_{2} \mathrm{O}_{3}, \mathrm{Zr}$ phosphates) are also being explored, all of which have been seen to provide modest to exceptional friction reduction in devices. By expanding the library of solid lubricants which may be employed at sliding interfaces, lubrication of a broader range of materials in a wider range of environments may be achieved, and single layer studies of these materials 
have begun to expand. Oddly enough, by considering lubrication with a single layer, the weak interlayer interactions that have largely defined their lubricity are no longer present, and interactions between the layered material and the substrate dominate. Rather, interest in single layers is largely motivated by the interaction anisotropy that renders such layered materials relatively chemically inert, as well as the high mechanical strength of these materials, which together offer a means to chemically and mechanically modify a surface with only a single atomic layer of material.

That is not to say that these studies are not interesting or useful in their own right in terms of solid lubrication. The ability to study the frictional characteristics of single and few layered samples of these materials makes it possible to isolate the effects of the interface between the lubricant and the substrate, which is more challenging when the majority of sliding occurs between the layers. These interactions become increasingly important in demanding nanoscale applications where the most effective lubrication with the least amount of material is essential, and in these cases, the lubricant-substrate interface will have increasing influence on the mechanical, dissipative, and chemical properties of the layered lubricant, it is therefore essential that these interactions, and the factors that influence them, be understood.

There are effectively two interfaces that must be understood to characterize and optimize the frictional characteristics or layered materials. First, and probably foremost, is the interlayer interface, as the majority of interfaces lubricated with these materials will be protected by multiple layers and the majority of sliding will occur between the layers. Second, the interface between the substrate and the lubricant, which becomes increasingly important as the number of layers at the interface is reduced. Factors like nanoscale morphology and chemistry are perhaps the most important, raising issues of film conformity that leads to behaviors like wrinkling and puckering that create increased real area of contact and additional dissipation pathways that influence the frictional characteristics of these materials.

This review focuses on three critical areas of 2D lubricant materials. First, the influence of interlayer interactions, as studied through a variety of computational methods, are discussed, as these interactions 
form the foundation of layered material lubricity in the majority of applications. Next, studies examining substrate-lubricant interactions, facilitated by the isolation of single and few layer graphene and other layered materials, are discussed, emphasizing this new area of research in layered materials and exploring factors like substrate morphology, interaction strength, and the sliding environment. Finally, studies seeking to apply 2D nanomaterials in sliding contacts are discussed, exploring their incorporation in lubricant fluids as nanoparticles and platelets in order to evaluate the relevant challenges in the incorporation of these materials as lubricants and lubricant additives.

\section{Interlayer Interactions in Layered Materials}

Computational approaches provide an excellent means to study the underlying mechanisms governing the friction of layered materials. Application of these techniques largely focus on two key aspects: contributions to friction from structural features that are either too small to resolve or inaccessible to experiment like atomic scale roughness [34], defects, wrinkling [35], and step edge effects [36], and the role of interlayer interactions in the response of sliding materials. Perhaps the most
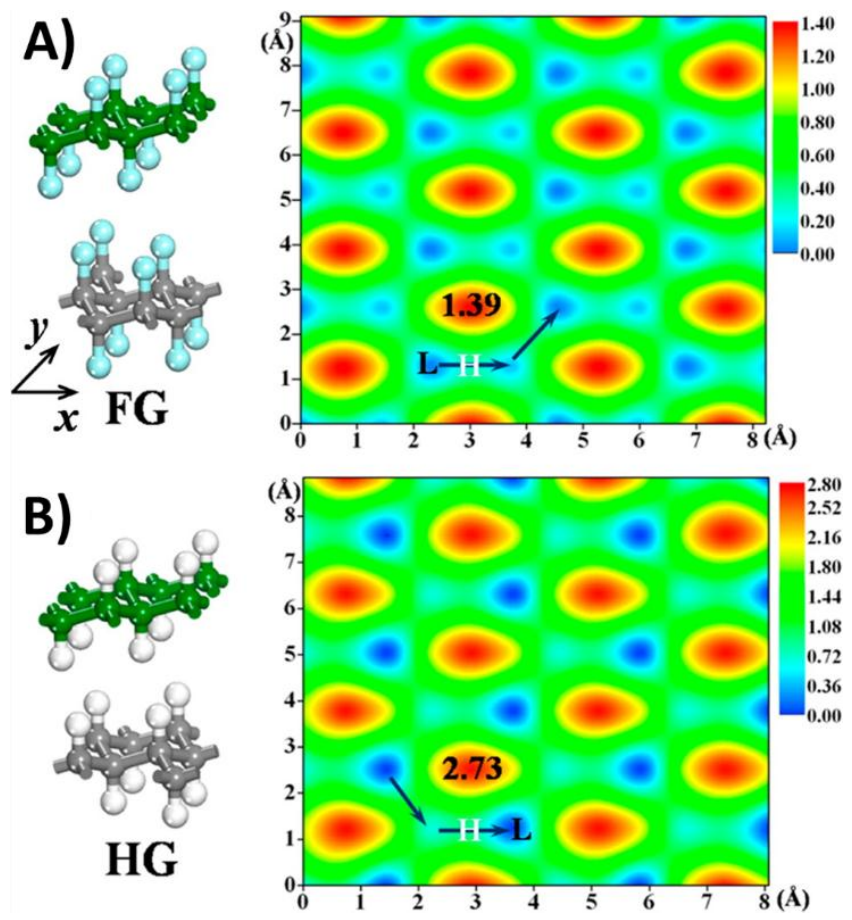

Figure 2. Models of fluorinated (A) and hydrogenated (B) graphene (FG and HG respectively), where grey atoms indicate carbon, blue fluorine, and white hydrogen. Corresponding potential energy surfaces are shown, with the favored path of sliding indicated. The highest and lowest energy points on these sliding pathways are denoted $\mathrm{H}$ and L respectively. Reproduced with permission from Ref. [37]. Copyright 2013, American Chemical Society. 
appealing feature of layered materials is the general weakness of their interlayer interactions, and their superlubricity is largely attributed to variations in these interactions. These contributions to the friction response are challenging to probe in single asperity measurements, and though they are present in macroscopic sliding contacts, they cannot be easily isolated. Computational approaches therefore provide the best means available to study the role of interlayer interactions and the mechanisms that give rise to superlubricity of layered materials.

The potential energy corrugation is a fundamental parameter in determining the magnitude of the shear strains that originate during sliding, and consequently influences the magnitude of the friction force. Determination of the interlayer potential requires only a single unit cell geometry, a size that is easily accessible by density functional theory (DFT) [38, 39], and this approach has been used to investigate the interlayer interactions of molybdenum disulfide [40, 41], graphite [39], and hexagonal boron nitride [38], and derivatives [42, 43]. Modified DFT functionals with dispersion corrections must typically be employed due to the weak interactions between sheets, though the shape of the potential can be obtained by applying pressure to the system, such that Pauli repulsion dominates the interlayer interaction [44]. Sample potential energy surfaces of hydrogenated and fluorinated graphene are depicted in Figure 2, showing the maximal potential energy and the most favorable sliding pathway, and a summary of the potential variations in common layered materials is provided in Table 1.

\begin{tabular}{|l|l|l|l|}
\hline Material & $\mathbf{U}_{\max }-\mathbf{U}_{\min }(\mathbf{m e V} /$ atom) & $\begin{array}{l}\mathbf{U}_{\max }-\mathbf{U}_{\min } \text { (meV/atom) } \\
\text { (Free sliding path) }\end{array}$ & Method[Ref.] \\
\hline Graphene & 10 & $2-3$ & DFT-PBE-D[39] \\
\hline h-BN & 30 & $3-4$ & $\begin{array}{l}\text { DFT-B3LYP+TS- } \\
\text { vdW[38] }\end{array}$ \\
\hline Graphene/h-BN & 20 & 15 & DFT-PBE-vdW[42] \\
\hline F-Graphene & 1.4 & 0.3 & DFT-PBE-D[37] \\
\hline H-Graphene & 2.7 & 1 & DFT-PBE-D[37] \\
\hline MoS $_{2}$ & 30 & 10 & DFT-PBE-D[41] \\
\hline
\end{tabular}

Table 1. Compilation of available sliding energy surface corrugation maxima and variation along the free sliding path measured at the equilibrium separation of the layers, expressed in terms of the potential energy difference between points of highest $\left(\mathrm{U}_{\max }\right)$ and lowest $\left(\mathrm{U}_{\min }\right)$ potential energy along the sliding path. 

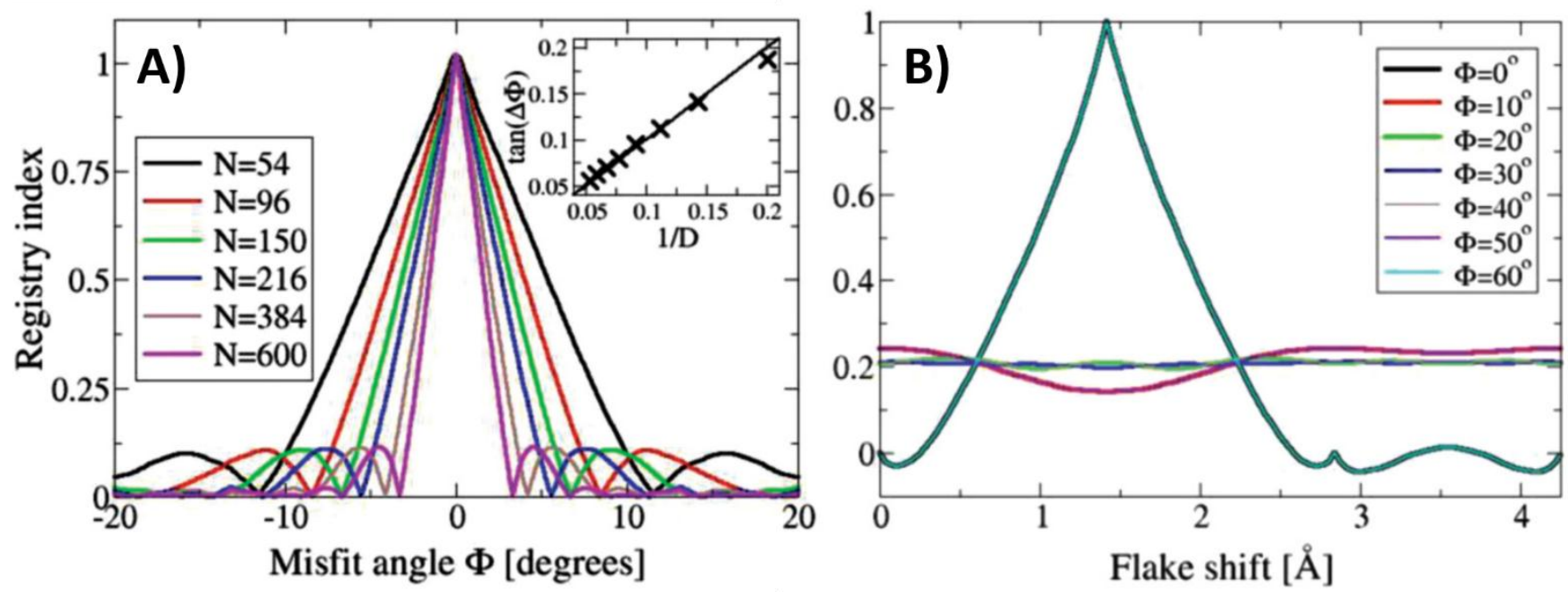

Figure 3. Variation of registry index as a function of flake size, expressed in terms of $\mathrm{N}$ carbon atoms, and misfit angle (A), and variation in registry index during sliding for different misfit angles $(\Phi)$ between sheets (B). The dramatic reduction in registry index variation results in dramatic reductions in friction. Adapted from Ref [45]. Copyright 2012 American Physical Society.

Interestingly, hydrogenated and fluorinated graphene exhibit the lowest potential corrugation, suggesting that they would exhibit the lowest friction of these materials when sliding occurs between layers. To date, however, only Atomic Force Microscopy (AFM) measurements of friction across the modified graphene surface, rather than sliding between layers of the material, have been performed. In these cases, greater friction than that observed for pristine graphite was observed [46], and atomic roughness of the modified graphene sheet [47] or contributions due to increased out-of-plane stiffness[46] have been proposed as a mechanism for the greater friction response under these conditions.

Breaking the periodicity of a layered system dramatically reduces the potential corrugation of interlayer sliding. The effect is difficult to determine by ab initio methods, but through application of the registry index developed by Hod et al., the effect is clearly illustrated [48]. The registry index is a normalized result, varying from 0 to 1 , which describes the favorability of a given stacking configuration. A value of 0 corresponds to two layers at their equilibrium position, consistent with the crystal structure of the material, while 1 corresponds to the most unfavorable position. For example, graphene varies from 

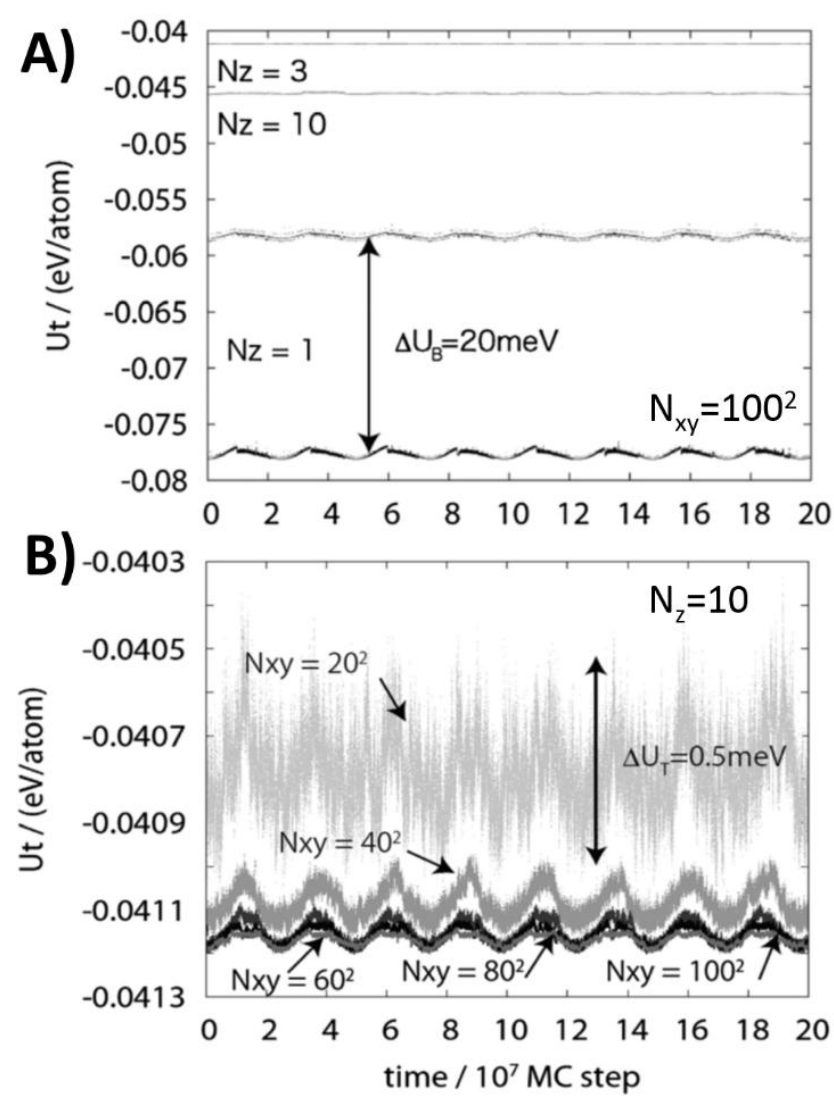

Figure 4. Thermal fluctuations in the potential energy $\left(\mathrm{U}_{\mathrm{T}}\right)$ for varying numbers $\left(\mathrm{N}_{\mathrm{z}}\right)$ of stacked graphene flakes (A) and sheets of varying size, expressed in terms of the number of carbon atoms represented in the sheet $\left(\mathrm{N}_{\mathrm{xy}}\right)$. Large fluctuations for the case $\mathrm{N}_{\mathrm{z}}=1$ were observed because the single sheet was force to oscillate between two stable points dictated by the upper and lower fixed sliders, the difference in these two states denoted by $\Delta U_{B}$. Adapted with permission from Ref. [49]. Copyright 2012, RSC Publishing.

0 for the $\mathrm{AB}$ crystalline structure to 1 for the AA structure in which all atoms overlap, while isoelectronic h-BN varies from 0 for the AA' stacking structure to 1 for AA stacking. Through proper tuning of the parameters used to determine the registry index, it scales roughly with the potential energy, and can be more easily calculated for large, aperiodic systems. Figure 3A depicts the registry index as a function of mismatch angle in graphene for a range of flake sizes. Large flakes require a smaller rotation to become effectively incommensurate [45], but more energy is required to achieve this state. Figure 3B depicts the variation in the registry index for different misfit angles, demonstrating dramatic reduction in the variation of the RI, and a corresponding reduction in the friction response has been observed for graphitic systems 
$[2,45,50]$. This is not a particularly surprising result, sliding of commensurate crystals is generally observed to produce a very high friction response due to the large area and numerous, constructive interactions involved. Layered materials offer the weakest interlayer interactions conceivable but nevertheless, when commensurate, these interactions will scale with the size of the sliding interface which is unfavorable in terms of minimizing friction.

To maximize the lubrication benefit of layered materials it is thus necessary to identify the ideal conditions in which layers are able to escape the commensurate state. Using coarse-grained Metropolis Monte Carlo Brownian Dynamics, [51] Washizu et al. examined the dynamics of multilayer graphene sliding as a function of number of layers and sheet size to determine the likelihood of "thermal escape," i.e. the slipping of graphene flakes out of the commensurate state due to thermal energy [49]. In this work, they examined the dynamics of graphene sheets sliding on an infinite graphite plane, and the variations in potential energy of these sliding simulations are depicted in Figure 4A and 4B as a function of layer count and sheet size respectively. They found that single sheet motion on an infinite graphene plane is highly restricted, and these restrictions result in large oscillations of the system energy that can give rise to greater friction. Flake size also dictates the magnitude of the interactions. In the smallest flakes studied $(\mathrm{r}=2$ $\mathrm{nm}$ ), thermal fluctuations dominate the variation in potential energy. For larger flakes, thermal fluctuations are more muted because the total interaction energy scales with flake size, but give rise to more stable potential energy profiles that would yield low friction. For the largest flakes studied ( $r=90$ $\mathrm{nm}$ ), however, thermal energy was insufficient to dislodge the flakes from the commensurate state, and their highly restricted motion would similarly give rise to greater friction. These results indicate that the most lubricating materials would consist of both multi-layered, and small grained structures. 


\section{Graphene: 2D or not 2D? That is the question.}

Graphene is one of the newest nanoscale carbon allotropes including carbon nanotubes and fullerenes. It is a 2D material with a wide array of interesting properties such as chemical stability, hydrophobicity, impermeability, excellent mechanical properties with a high in-plane elastic modulus [12], large out-ofplane flexibility, and low coefficient of friction [13-15]. These properties make it ideally suited for many applications, including the field of tribology, as a protective surface coating and friction modifier. There has been numerous experimental and theoretical studies devoted to understanding the structure-property relationship and modifications for improving the applicability of graphene to the field of tribology, but a broader fundamental understanding is still required.

Currently the frictional mechanisms for using $2 \mathrm{D}$ materials as friction modifiers has been found to depend on a variety of factors including the thickness of the graphene layers, the surface morphology and chemistry, and the fabrication method such as mechanical exfoliation, chemical vapor deposition, thermal decomposition, and reduction of graphene oxide. Some of the most recent work in this area is reviewed here and more thorough reviews, specifically on graphene, can be found elsewhere [52-54].

\section{Nanotribological Studies on Graphene}

To use graphene as a protective coating or friction modifier it must be interfaced with different substrates depending on the desired applications. Nanotribological experiments are key for understanding these interfacial interactions and the resulting fundamental friction mechanisms. Nanoscale studies using AFM investigated the friction of graphene on smooth and atomically rough surfaces and have found that the strength of graphene adhesion to the substrate largely depends on the morphology of the substrate [32, 33]. Experiments of exfoliated graphene on flat surfaces such as mica, $\mathrm{h}-\mathrm{BN}$, and graphite showed no 

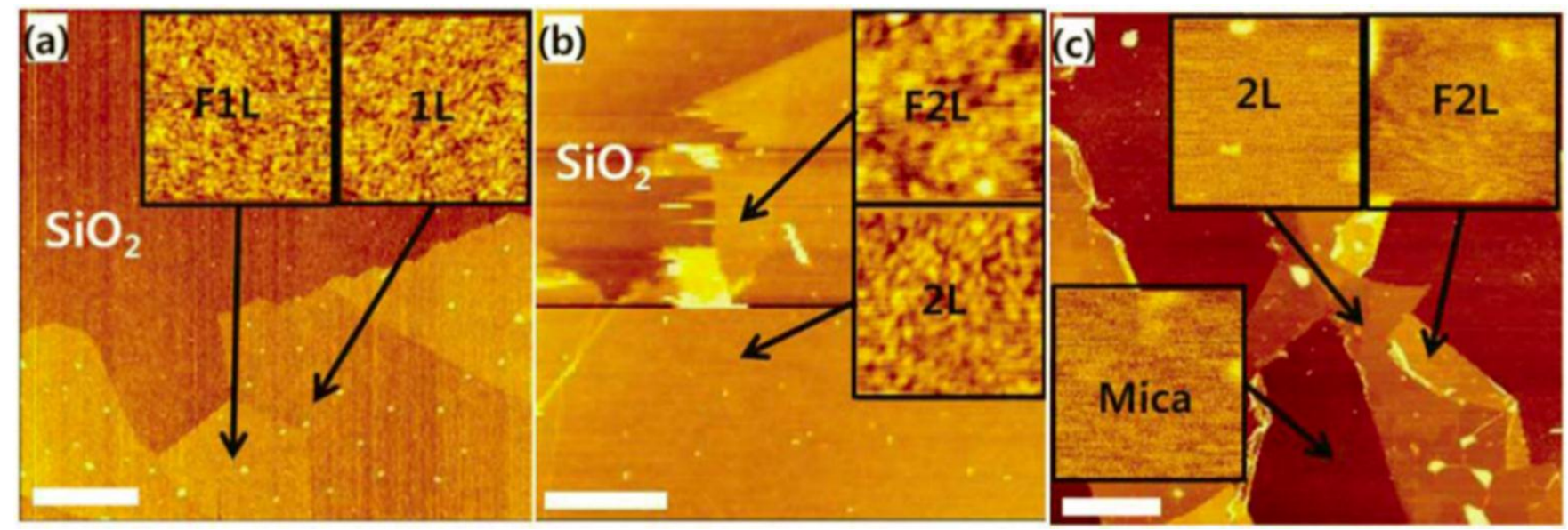

Figure 5. Topography images of (a) folded mono-layer graphene on $\mathrm{SiO}_{2}$, (b) folded bi-layer graphene on $\mathrm{SiO}_{2}$ and (c) folded bi-layer graphene on mica. Inset images acquired for the region indicated by black arrows have the same scan size of $500 \times 500 \mathrm{~nm}^{2}$ with identical z-scale (0 to $\left.1.23 \mathrm{~nm}\right)$. 'F1L' and 'F2L' represent folded mono- and bi-layer graphene, respectively. Scale bars, $2 \mu \mathrm{m}$. Reproduced from Ref. [32] with permission of The Royal Society of Chemistry.

layer dependence in the frictional properties, implying that graphene can reduce the friction as effectively as its bulk lamellar counterpart graphite when it makes good contact with the interface and has strong adhesion. However, on atomically rough surfaces such as $\mathrm{SiO}_{2}$, the intimate contact with the substrate is reduced which decreases the adhesive van der Waals forces between the substrate and graphene, inducing a "puckering" effect with friction as high as double that of bulk graphite. It known that the flexibility of graphene allows it to maximize its conformity to whatever substrate it is interfaced with, and by folding the flakes of graphene with an AFM tip, the morphology of the substrate was seen to be preserved as shown in Figure 5. Since the graphene maintained a rough configuration after folding from $\mathrm{SiO}_{2}$, the friction was still higher despite added thickness due to the decreased contact with the flat substrate. Hence, the adhesion to the substrate also depends on the morphology before and after transfer which should be considered in cases where graphene is processed after fabrication for transfer to other surfaces as a protective coating [32]. As the surface roughness transitions to the nanoscale, the balance of substrate morphology and graphene-substrate interactions plays a critical role in the friction observed, as it results 
in regions of conformed and unconformed states. For example, studies of graphene on silica nanoparticle films with controlled nanoscopic roughness (ca. $10 \mathrm{~nm}$ rms) show that the frictional properties strongly depended upon the relative adhesion between the AFM probe tip and the substrate, with suppression of the "puckering" effect, mentioned above, occurring when there is large surface roughness and low adhesion to the tip [55].

As most nanoscale studies have been done under ambient conditions the environmental dependence of friction and adhesion properties of graphene are also relevant. Under humid conditions, the adhesion [56] and friction forces generally decreased with lower humidity due to reduction of the capillary force but similar trends, such as the puckering effect, are still observed even under dry nitrogen and vacuum $[33,57]$. The effect of liquid environment on the adhesion and friction forces of few layer graphene has been found to be different from ambient studies in that the measured forces also depend on the polarity of the solvent. It was seen that the friction coefficient and adhesion were similarly reduced in liquid environment due to the loss of the water meniscus, which dominates the adhesion under ambient conditions. However, using ultrasonic force microscopy, the few layer graphene was observed to be more relaxed in liquid environment with increased contact to the interface. The lowest adhesion was measured in the nonpolar dodecane solvent, although higher friction was measured in dodecane over water. This was attributed to a $\sim 2 \mathrm{~nm}$ thick solid-like cushion structure between the tip and few layer graphene in dodecane and it was concluded to be responsible for the increased friction [58].

Although mechanical exfoliation is suitable for fundamental nanoscale studies, it is not sufficient for bulk processing and over the years the quality of other fabrication methods such as CVD of graphene has improved which is more suitable for applications $[59,60]$. However, since the tribological properties of graphene depend on the substrate it is fabricated on and transferred to, fundamental studies of the frictional properties of graphene on these substrates are also important. A recent study of CVD graphene grown on 
copper foil demonstrated a layer dependence of friction between bilayer and single layer graphene. The puckering effect was again observed and found to have a strong dependence on the normal force and sliding history with larger deformations observed at larger loads indicating a weak interaction with the underlying copper substrate [61]. In the case of CVD graphene grown on $\mathrm{Ni}(111)$, it was found that the graphene-Ni(111) interface has a strong interaction due to the covalent bonding character and is more rigid with respect to out-of-plane deformations with lower shear strength and work of adhesion compared to graphene on amorphous silica. [57]

Alternatively, graphene oxide films have also been considered as friction modifiers for MEMS devices. Since these films are known to have defects and contain an overall negative charge, the adhesion and friction are affected differently than pristine graphene coatings. This is especially important for devices containing electrical contacts, as it was found that when an AFM tip with a positive charge slides over the graphene oxide films, the charges are polarized creating an attractive electrostatic forces resulting in increased adhesion and friction until a saturation point is reached [62].

\section{Micro/Macrotribological Studies of Graphene}

Microscale tribological experiments, using a microtribometer, on graphene coated $\mathrm{SiC}-6 \mathrm{H}(0001)$ showed that graphene quickly delaminates from the substrate exposing the interfacial layer with twice the measured friction (Figure 6). This is due to the fact that the graphene is weakly adhered to the substrate and can easily be sheared off at points of high pressure such as step edges meaning that although graphene can reduce the friction, low loads and smooth surfaces are ideal to maintain the graphene coating [14].

Similar results were found when macroscale tribological experiments, using a high vacuum tribometer with a ball on disk contact geometry, were tested on few layer graphene/steel surfaces. A non-continuous film of graphene flakes (25\% coverage) was deposited on the substrate from an ethanol solution. The coefficient of friction was stabilized around 0.18 , approximately six times lower than the uncoated steel 

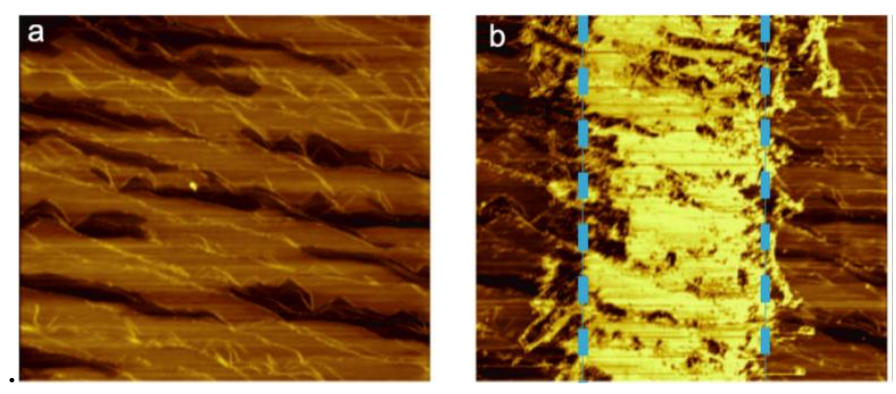

Figure 6. AFM friction maps of the graphene-covered $\mathrm{SiC}$ surface before (a) and after (b) a microtribological experiment. Dark areas indicate low and bright areas high friction. The images have a width of $20 \mu \mathrm{m}$, the color scale ranges from $1 \mathrm{nN}$ to $12 \mathrm{nN}$. The dashed lines in (b) indicate the estimate of the contact diameter. Reprinted with permission from Ref. [14]. Copyright 2013, Elsevier.

surface showing good friction reduction even under dry nitrogen environment unlike bulk graphite samples. Analysis of the wear tracks from long sliding cycles revealed a reduction in wear by two orders
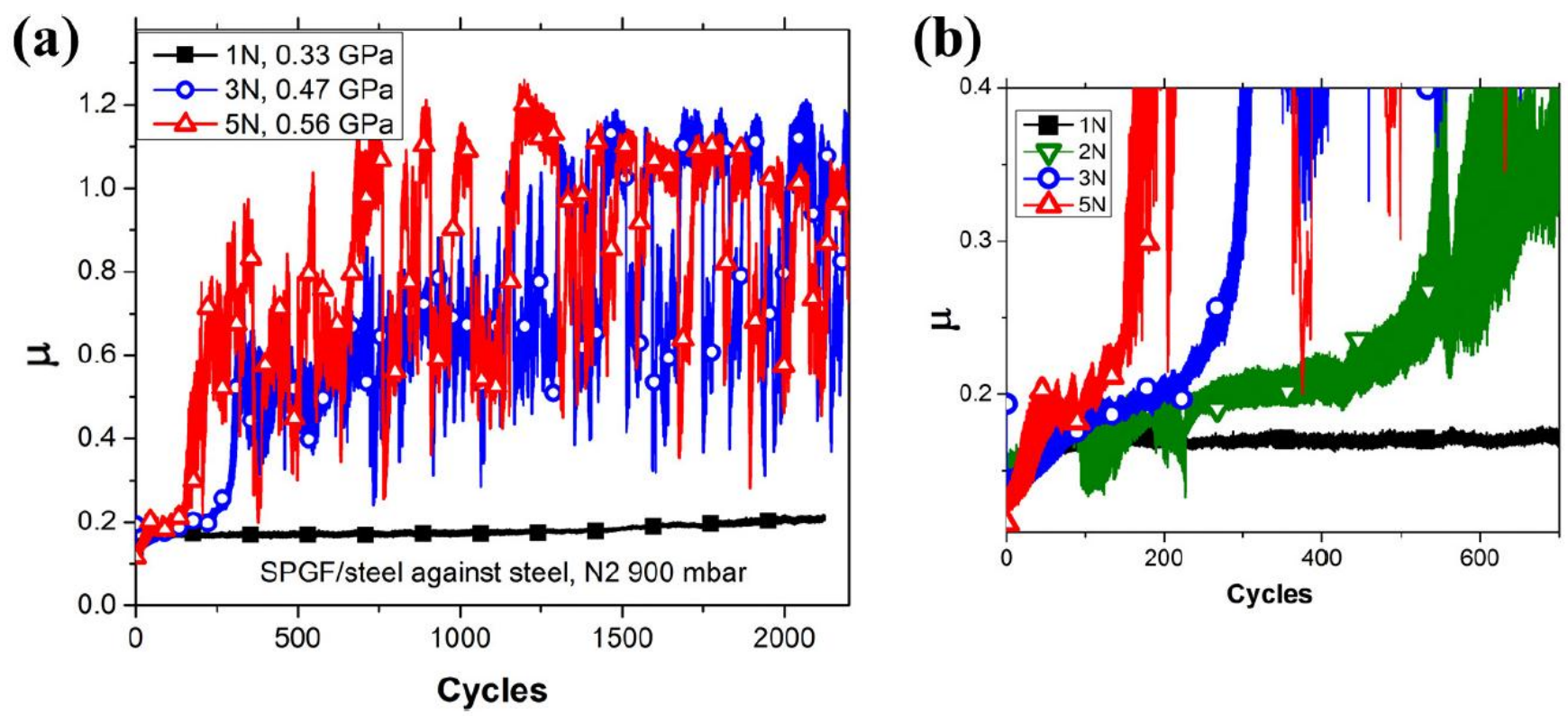

Figure 7. Coefficient of friction for steel with solution processed graphene flakes (SPGF) under different loads (a) for 2000 cycles; (b) represents magnified view for initial cycling period. The average Hertz contact pressure is presented for every applied load. Reprinted with permission from Ref. [63]. Copyright 2013, Elsevier. 


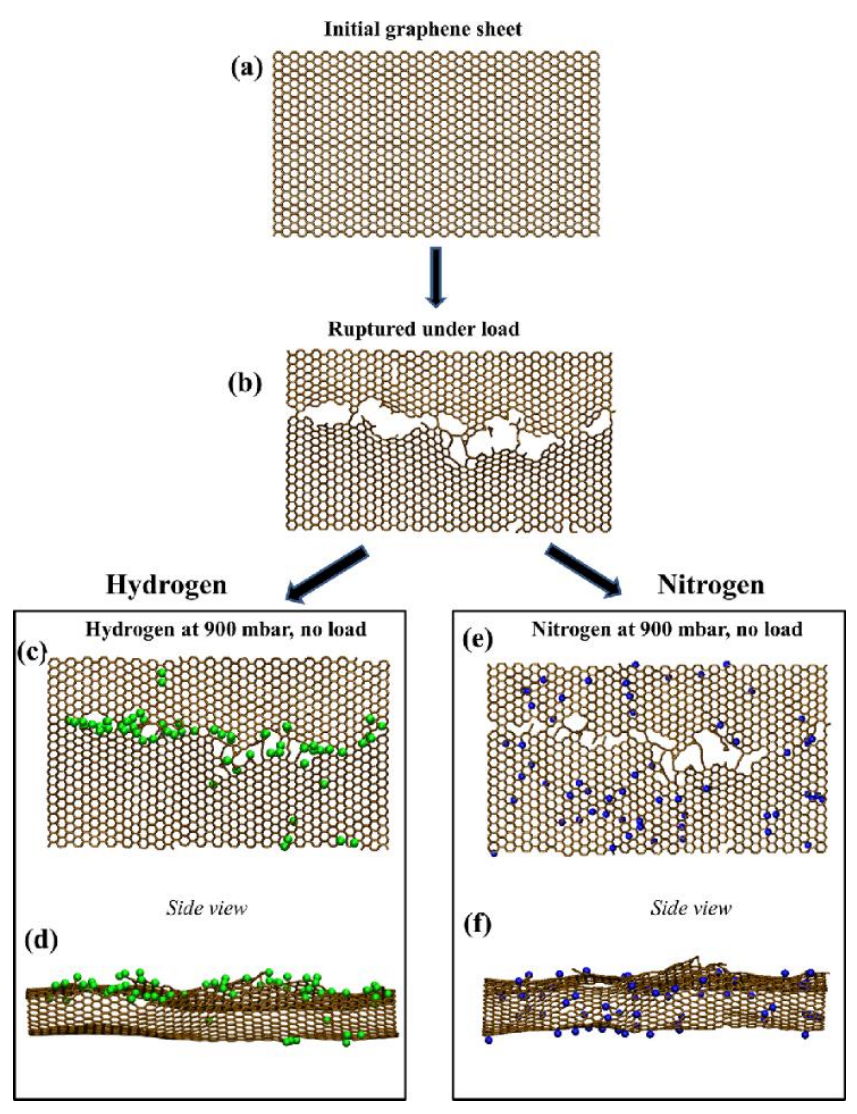

Figure 8. Graphene modification under different gas environments. Hydrogen environments afford capping of dangling bonds (hydrogens in green), while nitrogen atmospheres (nitrogen in blue) do not and show not preferential bonding to defect sites. Reprinted with permission from Ref. [64]. Copyright 2014. Wiley-VCH

of magnitude on the graphene coated substrates compared to the bare steel. Load studies from 1 - $5 \mathrm{~N}$, shown in Figure 7, indicated better durability under low loads lasting the full 2000 cycles, while at higher loads the interface suffered immediate high friction and wear due to the removal of the graphene layers from the track [63].

Most recently Berman et al. have shown that stabilization of graphene films can be observed when the gaseous environment in which the wear is occurring is controlled [64]. From a combination of experimental and computational studies they demonstrated that when wear experiments were conducted in hydrogen versus nitrogen environments, wear could be dramatically impeded when in hydrogen. From 
the computational studies it has been proposed that under hydrogen environments, "healing" of defects in the graphene can be achieved as the hydrogen will passivate dangling bonds and defects in the film, whereas in nitrogen, no reaction with the graphene films occurs (Figure 8). The hydrogen is also purported to suppress oxide formation in the steel surfaces studied.

Along with other large scale fabrication techniques, graphene oxide presents an alternative solution based method for deposition of graphene films. Graphene oxide (GO) may be reduced to form graphene as well as offer alternative hybrid composite films for tribological applications due to its chemical reactivity and good solvent dispersion. Macroscale tribological studies between a sintered tungsten carbide ball and stainless steel substrate have shown that 1 wt. $\%$ of graphene oxide in water can sustain low friction up to 60,000 cycles with a friction coefficient as low as 0.05 (Figure 9), likely due to the continuous replenishment and adsorption of flakes from the solution [65].

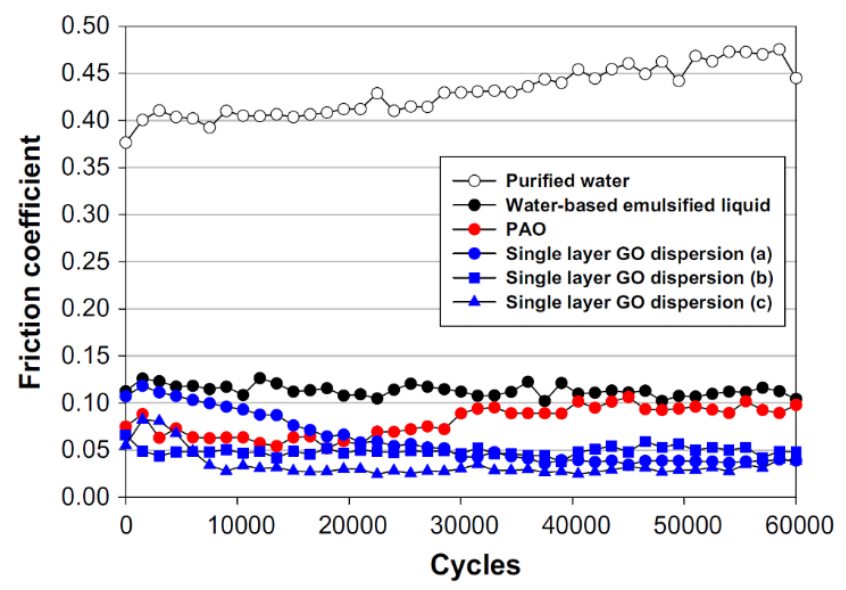

Figure 9. Variations of friction coefficients lubricated with purified water, the water-based emulsified liquid, polyalphaolefin (PAO), and the graphene oxide (GO) dispersion, as a function of friction cycles, between the WC ball and stainless steel flat plate under the load of $1.88 \mathrm{~N}$. Reprinted with permission from Ref. [65]. Copyright 2013, Elsevier.

The chemically reactive functional groups on graphene oxide (typically, $-\mathrm{OH},-\mathrm{COOH}$ and epoxide) have also been utilized to form thin films by which graphene is covalently bound to the substrate to overcome 
its intrinsic adhesion and debonding from substrates where graphene is only stabilized by van der Waals forces. These films can then be interfaced with other mobile lubricants as friction reducers and the immobilized graphene layers as the wear reducers. This idea has already been proven effective where graphene oxide was immobilized on substrates layered with multiply-alkylated cyclopentanes and $\mathrm{C}_{60}$ and microtribological tests using a ball-on-plate tribometer were performed $[66,67]$. In both cases the synergistic effect of the hybrid films allowed for good friction and wear resistance due to the replenishing of the mobile lubricants and rolling of the $\mathrm{C}_{60}$ combined with the mechanical resistance of the graphene oxide. Additionally, graphene flakes have been utilized as filler additives to form nanocomposites and enhance the matrix properties including the friction and wear resistance. In one recent example, modified graphene/polyimide nanocomposites prepared through surface modification of the graphene and in situ polymerization showed enhancements in thermal stability, electrical conductivity, mechanical and tribological properties. It can be seen in Figure 10, when 2 wt. \% modified graphene was used in the nanocomposites the friction coefficient was reduced by $12 \%$ and the wear rate decreased by $95 \%(20 \mathrm{x}$ increase in wear resistance) compared to polyimide without the modified graphene [68].

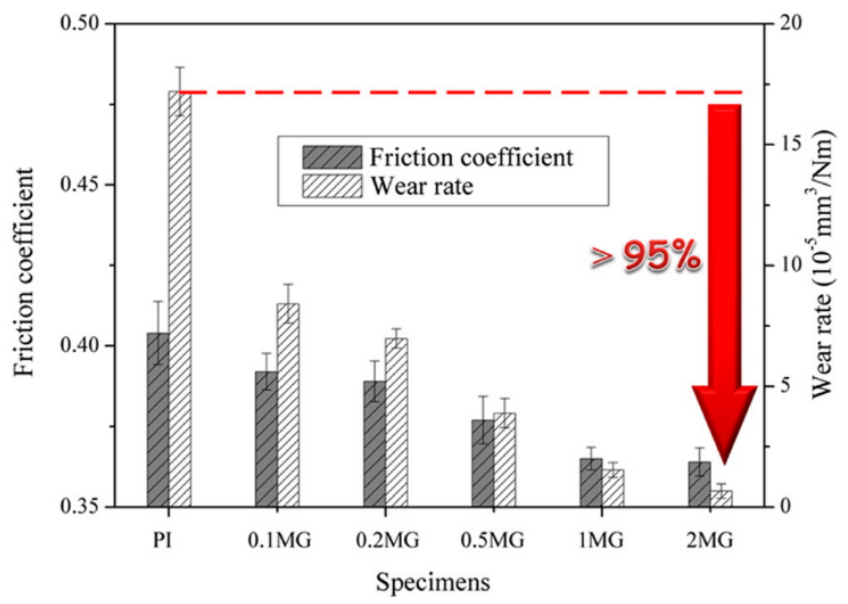

Figure 10. Friction coefficient and wear rate of neat polyimide (PI) and various weight percent values of modified graphene (MG) /polyimide specimens. Reprinted with permission from Ref. [68]. Copyright 2013, American Chemical Society. 


\section{Layered Nanomaterials}

It is clear that graphene is currently a hot topic in tribology research, but it has also opened up interest in similar 2D nanomaterials as well. Other well-known lamellar materials such as h-BN, $\mathrm{MoS}_{2}$, and $\mathrm{WS}_{2}$ are now starting to be investigated as single and few layer coatings. Recently it was shown, using mechanical exfoliation, that these materials exhibit a similar nanoscale friction mechanism despite having different electronic compositions and properties. As in the case of graphene, thin $2 \mathrm{D}$ single and few layer sheets of h-BN, $\mathrm{NbSe}_{2}$, and $\mathrm{MoS}_{2}$ also show the characteristic "puckering effect" which results from weak adhesion to the silica substrate and is a universal concern for materials of this type [33]. Although this research highlights the possibility of non-graphene 2D coatings, little work has been done to explore and take advantage of the unique properties these materials have to offer. Single layer hexagonal boron nitride has received slightly more attention recently when the tribological properties of CVD grown single layers on copper were investigated. The performance of the monolayers showed excellent friction reduction (up to $40 \mathrm{x}$ lower) as compared to the bare copper surface [69]. It is notable that in addition to friction modification, both CVD graphene and hexagonal boron nitride also have the ability to protect metallic interfaces against oxidation [70, 71] however, hexagonal boron nitride can sustain much higher temperatures before oxidizing, making it more desirable for high-temperature applications than graphene.

Unlike graphene where numerous fabrication methods have been explored and optimized these alternative materials are typically sputter coated or produced in the form of nanoparticles. Nanoparticles have been studied for many years and are of interest due to their ability to be used as solid lubricants, additives in liquid lubricants such as oils, and incorporated into nanocomposites. When combined with other friction modifiers, the nanoparticles offer unique advantages in that they are more environmentally friendly while maintaining good friction and wear reduction. In particular, when materials such as transition metal dichalcogenides $\left(\mathrm{MoS}_{2}, \mathrm{WS}_{2}\right)$, which are well known for their friction reducing properties 
in bulk form, are scaled down to the nanoscale the good frictional properties are maintained but the mechanisms are different when used as nanoparticles as compared to 2D lamellar sheets.

The origin of these excellent nanoparticle tribological properties has been extensively studied over the years and hypothesized to consist of three possible mechanisms including rolling, sliding, and

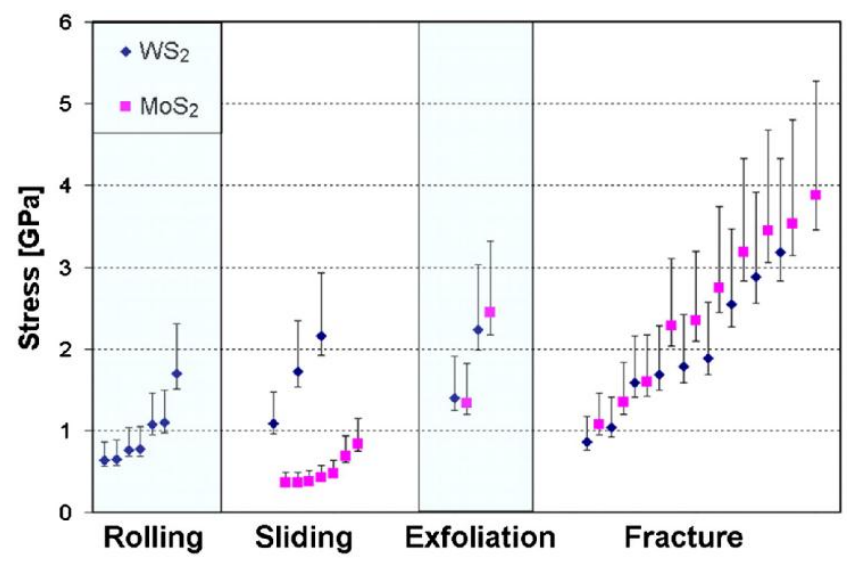

Figure 11. The stress values in ascending order, for the three main mechanisms and the fracture stress of IF-WS 2 and IF-MoS $\mathrm{S}_{2}$ ' IF' refers to inorganic fullerene-like nanoparticles of tungsten disulfide $\left(\mathrm{WS}_{2}\right)$ and molybdenum disulfide $\left(\mathrm{MoS}_{2}\right)$. Reprinted with permission from Ref [72]. Copyright 2011, National Academy of Sciences.
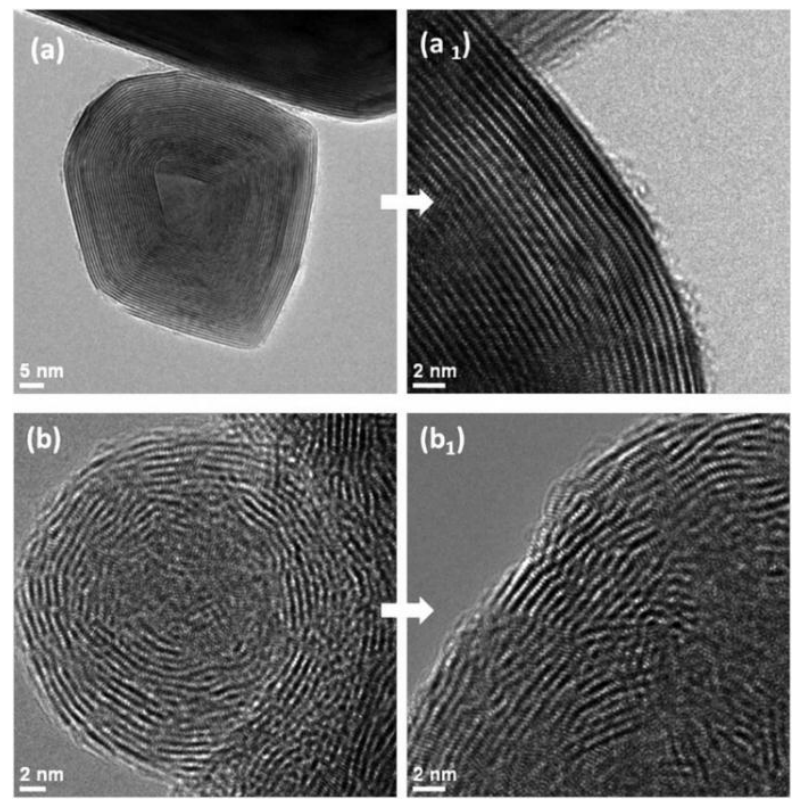

Figure 12. High-resolution transmission electron microscope images of (a and $\left.\mathrm{a}_{1}\right)$ : perfectly crystallized IF-MoS ${ }_{2}$ nanoparticle (namely IF-MoS $\mathrm{S}_{2}-\mathrm{C}$ ) and (b and $\mathrm{b}_{1}$ ): poorly crystallized IF-MoS $\mathrm{S}_{2}$ nanoparticle (namely IF-MoS $2-A$ ). Reprinted with permission from Ref [73]. Copyright 2013, John Wiley \& Sons, Ltd. 
exfoliation. These mechanisms have since been observed by in situ transmission electron microscopy and scanning electron microscopy imaging $[72,73]$ coupled with a nanoindentor/nanomanipulator and were shown to depend on the normal and shear stress as summarized in Figure 11. It was found that rolling of the nanoparticles occurs at low shear rates and pressures, which depends on the spherical shape of the particle and their mechanical stability. The sliding of nanoparticles is also dependent on the mechanical strength as well as the amount of interfacial adhesion. In the case of exfoliation, which is typically the dominate mechanism, the outer layers of the nanoparticle delaminate under higher shear stress and pressures. The generated nanosheets are transferred to the contact region and align in the direction of sliding thereby reducing friction through formation of a tribofilm. The efficiency of the exfoliation was studied by comparing $\mathrm{MoS}_{2}$ nanoparticles with high and low degree of crystallinity (Figure 12).
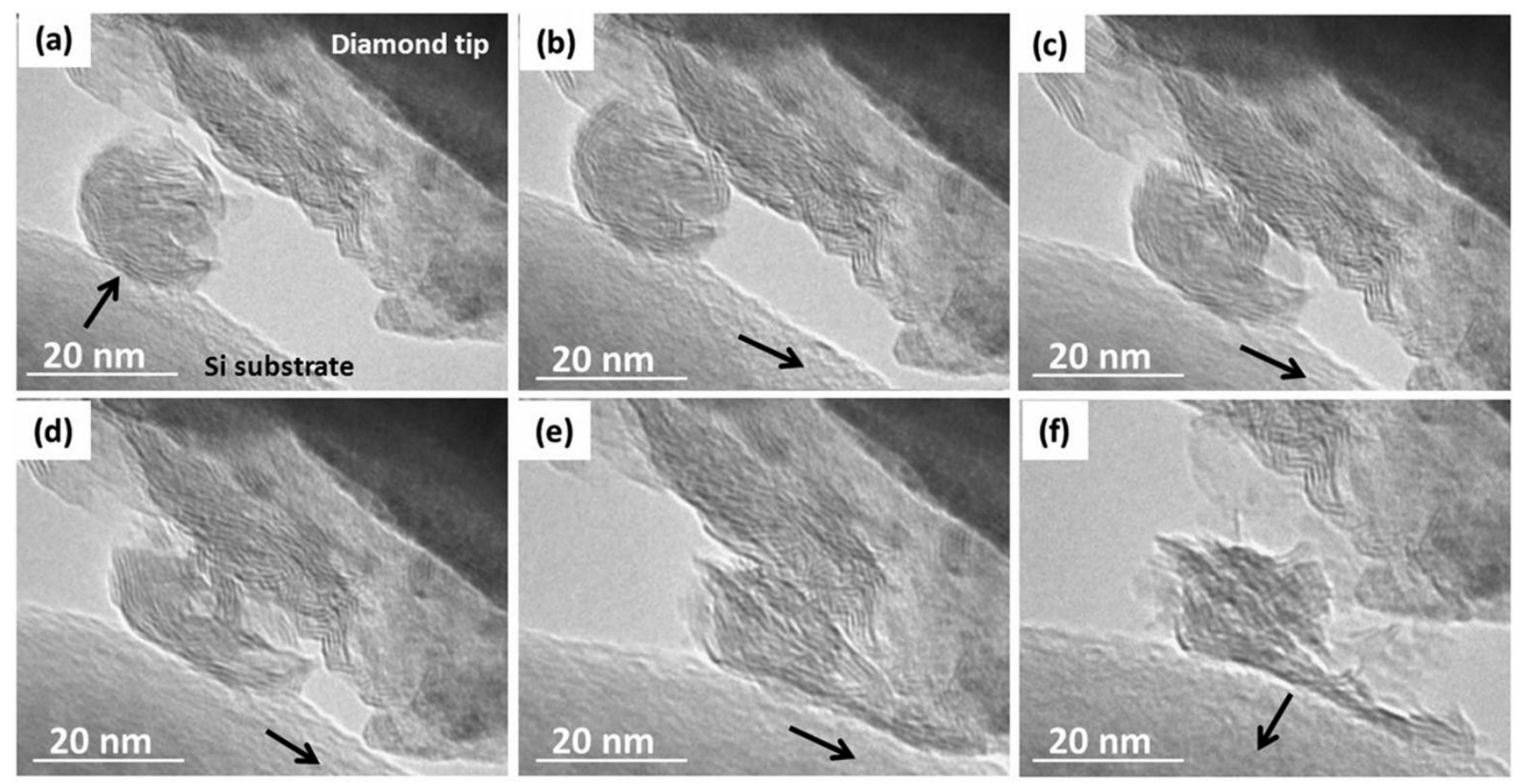

Figure 13. Image captures obtained from a video recorded during a sliding experiment carried out with a single poorly crystallized IF-MoS 2 nanoparticle (IF-MoS $2-\mathrm{A})$ demonstrating exfoliation. The black arrow on the Si substrate indicated the direction of the movement of the Si substrate. Reprinted with permission from Ref [73]. Copyright 2013, John Wiley \& Sons, Ltd. 


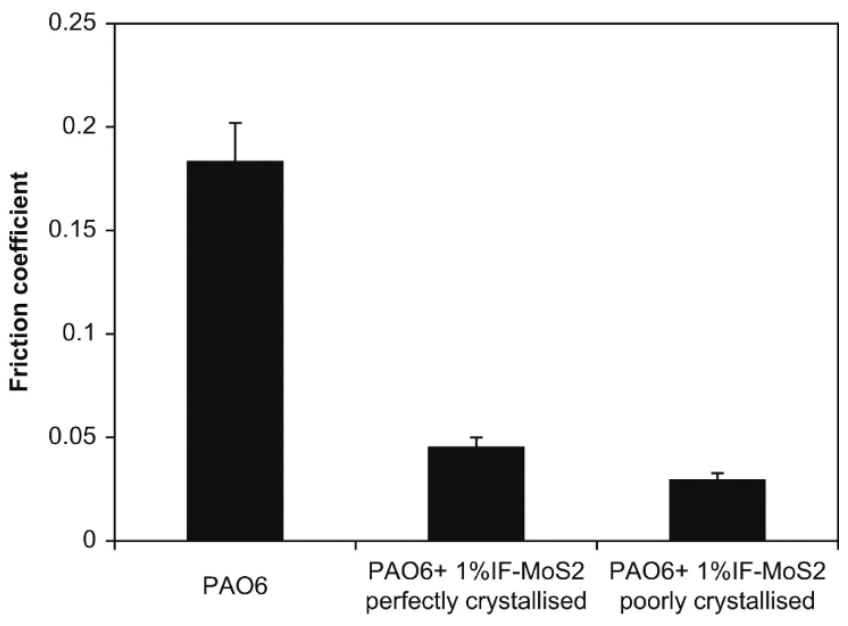

Figure 14. Friction coefficient of PAO6, PAO6 $+1 \mathrm{wt} \%$ of perfectly crystallized IF-MoS $\mathrm{M}_{2}$ and PAO6 +1 wt $\%$ of poorly crystallized IF-MoS 2 . Reprinted with permission from Ref [74]. Copyright 2012, Elsevier.

When there are more defects present in the nanoparticles, as in the case of low crystalline structures, the exfoliation is immediately observed in the high resolution transmission electron microscopy images (Figure 13), allowing for better friction reduction.

These in situ nanoscale studies help to visualize and correlate the friction mechanisms of nanoparticle additives to large scale friction studies. Macroscale ball-on-disc studies of 1 wt. $\% \mathrm{MoS}_{2}$ and $\mathrm{WS}_{2}$ nanoparticles in polyalphaolefin (PAO) on steel have shown that the additives are able to reduce the friction coefficient and wear due to formation of tribofilms [74, 75]. Analysis of the tribofilms revealed exfoliated lamellar sheets and embedded nanoparticles indicating the deformation and exfoliation of the nanoparticles was important for the friction reduction. The effect of crystallinity on the $\mathrm{MoS}_{2}$ nanoparticles was also investigated and the results were consistent with the TEM experiments with the poorly crystallized particles having a slightly better performance and the lowest friction coefficient as seen in Figure 14. The well crystallized particles were observed to have a continuous decrease in the coefficient of friction as well as an inhomogeneous tribofilm while the poorly crystallized particles generated a more 
homogeneous tribofilm and a faster reduction in the coefficient of friction, as expected, due to having more defects and easier exfoliation.

\section{Nanoparticle additives}

Nanoparticle additives are promising but not without limitations, especially since they are known to have issues with dispersibility and agglomeration that must be considered. However, these nanomaterials also offer a degree of tune-ability to overcome these drawbacks. For example, the morphology of the nanoparticles can take a variety of forms including spherical particles, hollow nano-vesicles, nanoplatelets, and nanotubes. In one comparison between $\mathrm{MoS}_{2}$ vesicles and platelets it was found that the vesicles performed better, both lowering the friction and improving wear resistance due to exfoliation which occurs at lower pressures ( $31 \mathrm{x}$ less than inorganic fullerene like nanoparticles). The poor performance of the nano-platelets was attributed to their higher tendency to precipitate as compared to the vesicles, which inhibits their ability to enter the contact and reduce friction [76]. In another case, the composition of the $\mathrm{MoS}_{2}$ particles was studied by doping the inorganic fullerene-like nanoparticles with rhenium. The particles were observed to be more stable and less likely than the $2 \mathrm{H}-\mathrm{MoS}_{2}$ to precipitate, even at higher temperatures. This increased stability was likely due to reduced agglomeration and directly attributed to the effectiveness of the nanoparticles at lowering friction as compared to the base PAO oil [77].

Although it is clear that nanoparticles are an effective means of reducing friction and wear while offering some flexibility for tailoring the properties for optimal performance under various conditions, it is useful to have a direct comparison of particles with different sizes, morphologies and material composition. A recent study, using a ball-on-disc tribotester, has provided such a direct comparison between seven different particles including $\mathrm{MoS}_{2}$ nanotubes and platelets, $\mathrm{WS}_{2}$ nanotubes and inorganic fullerene like nanoparticles, 


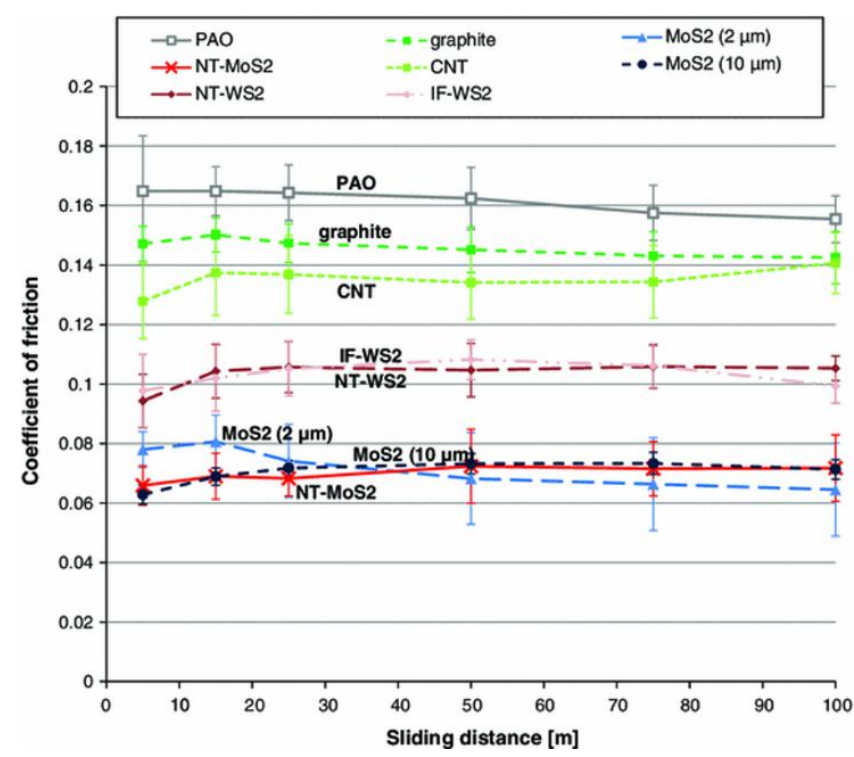

Figure 15. Coefficient of friction as a function of sliding distance for the base oil ('PAO') and the base oil with the addition of $5 \mathrm{wt} \%$ of various solid lubricant particles. Reprinted with permission from Ref [78]. Copyright 2014, Springer.

graphite platelets and multi-walled carbon nanotubes in PAO on steel. The evolution of the friction coefficients during sliding for the various materials can be seen in Figure 15. The coefficients of friction are closely related to the types of material with the $\mathrm{MoS}_{2}$ particles showing the best performance. Further wear loss experiments indicated the $\mathrm{MoS}_{2}$ and $\mathrm{WS}_{2}$ particles had the best wear reduction followed by the carbon nanotubes while the graphite platelets offered the least wear resistance. In this case, there was no dependence of the friction coefficients on size or morphology found, but it is noted that smaller particles have an intrinsic advantage in the ability to be more easily dispersed in the oil lubricant [78].

Akin to those studies carried out on graphene, $\mathrm{MoS}_{2}$ and h-BN, a host of other layered materials are being explored as friction modifiers. These include materials such as phosphates and layered oxides. Recently, Liang and co-workers have explored the use of $\mathrm{Zr}$ phosphate nano-platelets and yttrium oxide nanosheets $\left(\mathrm{Y}_{2} \mathrm{O}_{3}\right)$ as additives for lubricants as well as abrasive additives for processes such as chemomechanical planarization $[79,80]$. As with many of the above describe platelet systems, the $\mathrm{Zr}$ phosphate nano-platelets (on the order of $30 \mathrm{~nm}$ in thickness and $600 \mathrm{~nm}-1000 \mathrm{~nm}$ in length) can be 

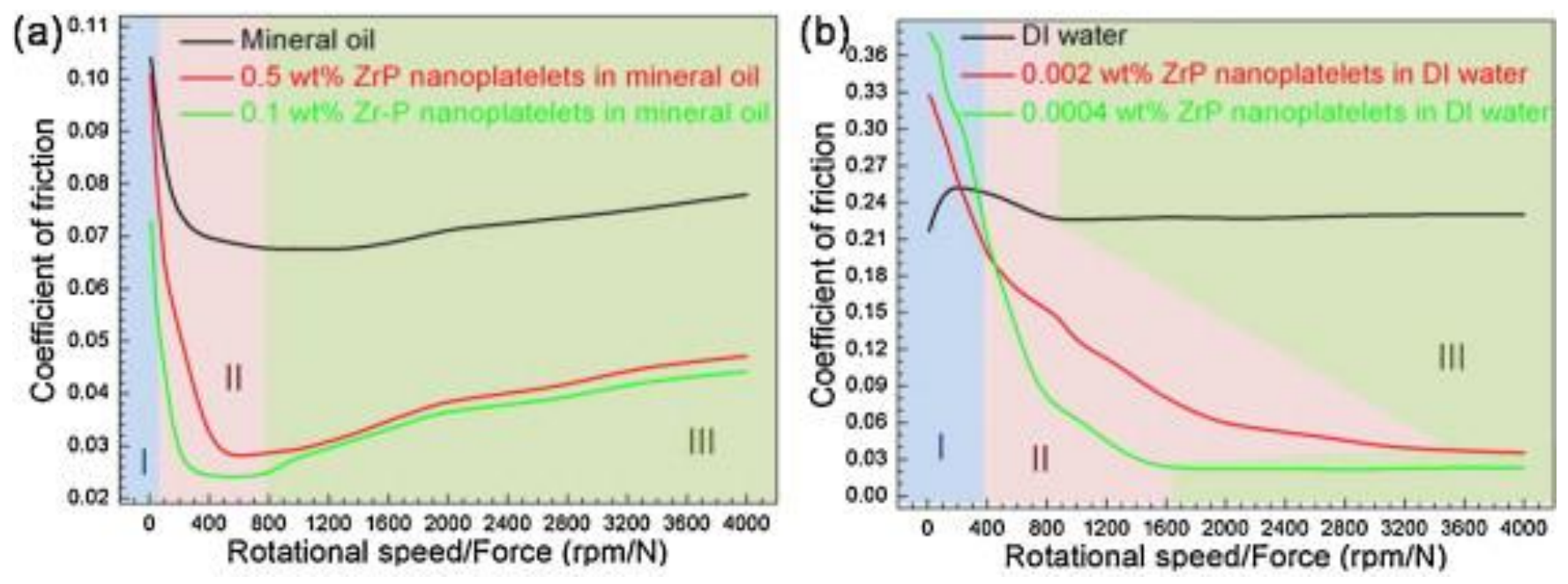

Figure 16. Stribeck curves of varying concentration of $\alpha$-zirconium phosphate (ZrP) nano-platelets in mineral oil (a) and water (b). Adapted with permission from Ref. [79]. Copyright 2014, Elsevier.

incorporated into oil or water based lubricants as an additive with loadings ranging from 0.0004 wt. \% 0.5 wt. $\%$, which showed decreases in friction from anywhere from $65 \%$ to $91 \%$ (Figure 16). These results are especially interesting as the use of materials such as $\mathrm{Zr}$ phosphate provides a green alternative to the production of lubricant additives as compared to materials such as $\mathrm{MoS}_{2}$, as these materials are basically clays. The resulting dramatic reduction of the coefficient of friction is attributed to two primary factors: the layering of the lubricant molecules along the platelet materials and the orientation of the platelets in the compressed sliding contact.

\section{Outlook}

The design of new friction modification and lubrication additives through the use of nanomaterials represents some unique and challenging opportunities across a range of systems. Clearly we see on the nanoscale the need to design interfaces that can achieve non-commensurate sliding conditions to minimize stick-slip. Computational studies can effectively guide the design of nanomaterials through chemical modification and combination, striving to minimize sliding strain and facilitate escape from the commensurate to incommensurate state. From the standpoint of additives, we can already see how the 
combination of nanomaterials in oils can offer new approaches to enhancing the friction reduction capabilities of typical liquid lubricants. Here advances in solubility, dispersability and particle degradation can play a major role in their efficacy. Similar solutions may be on the horizon for biological systems using materials such as graphene oxide, whose functionalization can make these materials water soluble allowing GO platelets to be incorporated with aqueous based lubricants for joint structures.

\section{Acknowledgements}

We gratefully acknowledge support from the National Science Foundation (CMMI-1436192) for our work on 2D nanomaterials for controlling friction and wear during the preparation of this review. 


\section{References}

[1] T. Spalvins, Thin Solid Films, 96 (1982) 17-24.

[2] M. Dienwiebel, G.S. Verhoeven, N. Pradeep, J.W.M. Frenken, J.A. Heimberg, H.W. Zandbergen, Phys. Rev. Lett., 92 (2004) 126101.

[3] S.J. Bull, Diamond Relat. Mater., 4 (1995) 827-836.

[4] L. Spanu, S. Sorella, G. Galli, Phys. Rev. Lett., 103 (2009) 196401.

[5] C. Busch, Solid Lubrication, Lubricants and Lubrication, Wiley-VCH Verlag GmbH \& Co. KGaA2007, pp. 694-714.

[6] K.S. Novoselov, A.K. Geim, S.V. Morozov, D. Jiang, Y. Zhang, S.V. Dubonos, I.V. Grigorieva, A.A. Firsov, Science, 306 (2004) 666-669.

[7] X. Du, I. Skachko, A. Barker, E.Y. Andrei, Nat. Nanotechnol., 3 (2008) 491-495.

[8] A.A. Balandin, S. Ghosh, W. Bao, I. Calizo, D. Teweldebrhan, F. Miao, C.N. Lau, Nano Lett., 8 (2008) 902-907.

[9] A.A. Balandin, Nat Mater, 10 (2011) 569-581.

[10] J. Renteria, D. Nika, A. Balandin, Applied Sciences, 4 (2014) 525-547.

[11] Z. Yan, D.L. Nika, A.A. Balandin, IET Circuits, Devices \& Systems, Institution of Engineering and Technology2015, pp. 4-12.

[12] C. Lee, X. Wei, J.W. Kysar, J. Hone, Science, 321 (2008) 385-388.

[13] K.-S. Kim, H.-J. Lee, C. Lee, S.-K. Lee, H. Jang, J.-H. Ahn, J.-H. Kim, H.-J. Lee, ACS Nano, 5 (2011) 5107-5114.

[14] F. Wählisch, J. Hoth, C. Held, T. Seyller, R. Bennewitz, Wear, 300 (2013) 78-81.

[15] A.L. Kitt, Z. Qi, S. Rémi, H.S. Park, A.K. Swan, B.B. Goldberg, Nano Lett., 13 (2013) 26052610.

[16] S. Stankovich, D.A. Dikin, G.H.B. Dommett, K.M. Kohlhaas, E.J. Zimney, E.A. Stach, R.D. Piner, S.T. Nguyen, R.S. Ruoff, Nature, 442 (2006) 282-286.

[17] Z. Chen, S. Berciaud, C. Nuckolls, T.F. Heinz, L.E. Brus, ACS Nano, 4 (2010) 2964-2968.

[18] M.D. Stoller, S. Park, Y. Zhu, J. An, R.S. Ruoff, Nano Lett., 8 (2008) 3498-3502.

[19] F. Xia, D.B. Farmer, Y.-m. Lin, P. Avouris, Nano Lett., 10 (2010) 715-718.

[20] J.S. Bunch, A.M. van der Zande, S.S. Verbridge, I.W. Frank, D.M. Tanenbaum, J.M. Parpia, H.G. Craighead, P.L. McEuen, Science, 315 (2007) 490-493.

[21] Q. Li, C. Lee, R.W. Carpick, J. Hone, Phys. Status Solidi B, 247 (2010) 2909-2914.

[22] T. Filleter, J.L. McChesney, A. Bostwick, E. Rotenberg, K.V. Emtsev, T. Seyller, K. Horn, R. Bennewitz, Phys. Rev. Lett., 102 (2009) 086102.

[23] T.-B. Ma, L.-F. Wang, Y.-Z. Hu, X. Li, H. Wang, Sci. Rep., 4 (2014) 3662.

[24] J.H. Seol, I. Jo, A.L. Moore, L. Lindsay, Z.H. Aitken, M.T. Pettes, X. Li, Z. Yao, R. Huang, D. Broido, N. Mingo, R.S. Ruoff, L. Shi, Science, 328 (2010) 213-216.

[25] W. Cai, A.L. Moore, Y. Zhu, X. Li, S. Chen, L. Shi, R.S. Ruoff, Nano Lett., 10 (2010) 16451651.

[26] J.-K. Lee, S. Yamazaki, H. Yun, J. Park, G.P. Kennedy, G.-T. Kim, O. Pietzsch, R. Wiesendanger, S. Lee, S. Hong, U. Dettlaff-Weglikowska, S. Roth, Nano Lett., 13 (2013) 34943500.

[27] T. Kase, T. Ogino, J. Phys. Chem. C, 117 (2013) 15991-15995.

[28] Q. Wu, Y. Wu, Y. Hao, J. Geng, M. Charlton, S. Chen, Y. Ren, H. Ji, H. Li, D.W. Boukhvalov, R.D. Piner, C.W. Bielawski, R.S. Ruoff, Chem. Commun., 49 (2013) 677-679.

[29] W. Zhu, T. Low, V. Perebeinos, A.A. Bol, Y. Zhu, H. Yan, J. Tersoff, P. Avouris, Nano Lett., 12 (2012) 3431-3436. 
[30] S.J. Goncher, L. Zhao, A.N. Pasupathy, G.W. Flynn, Nano Lett., 13 (2013) 1386-1392.

[31] S.p. Berciaud, S. Ryu, L.E. Brus, T.F. Heinz, Nano Lett., 9 (2008) 346-352.

[32] D.-H. Cho, L. Wang, J.-S. Kim, G.-H. Lee, E.S. Kim, S. Lee, S.Y. Lee, J. Hone, C. Lee, Nanoscale, 5 (2013) 3063-3069.

[33] C. Lee, Q. Li, W. Kalb, X.-Z. Liu, H. Berger, R.W. Carpick, J. Hone, Science, 328 (2010) 7680.

[34] D. Yalin, J. Phys. D: Appl. Phys., 47 (2014) 055305.

[35] Z. Ye, C. Tang, Y. Dong, A. Martini, J. Appl. Phys., 112 (2012) 116102.

[36] Z. Ye, A. Otero-de-la-Roza, E.R. Johnson, A. Martini, Appl. Phys. Lett., 103 (2013) 081601.

[37] L.-F. Wang, T.-B. Ma, Y.-Z. Hu, H. Wang, T.-M. Shao, J. Phys. Chem. C, 117 (2013) 1252012525.

[38] I. Leven, I. Azuri, L. Kronik, O. Hod, J. Chem. Phys., 140 (2014) 104106.

[39] M. Reguzzoni, A. Fasolino, E. Molinari, M.C. Righi, Phys. Rev. B: Condens. Matter, 86 (2012) 245434.

[40] T. Liang, W.G. Sawyer, S.S. Perry, S.B. Sinnott, S.R. Phillpot, Phys. Rev. B: Condens. Matter, 77 (2008) 104105.

[41] G. Levita, A. Cavaleiro, E. Molinari, T. Polcar, M.C. Righi, J. Phys. Chem. C, 118 (2014) 13809-13816.

[42] Z. Xiaoyang, L. Linyang, Z. Mingwen, J. Phys.: Condens. Matter, 26 (2014) 095002.

[43] G. Smith, N. Modine, U. Waghmare, E. Kaxiras, J. Comput. Aided Mater. Des., 5 (1998) 61 71.

[44] T. Liang, W.G. Sawyer, S.S. Perry, S.B. Sinnott, S.R. Phillpot, Physical Review B, 77 (2008) 104105.

[45] O. Hod, Phys. Rev. B: Condens. Matter, 86 (2012) 075444.

[46] S. Kwon, J.-H. Ko, K.-J. Jeon, Y.-H. Kim, J.Y. Park, Nano Lett., 12 (2012) 6043-6048.

[47] D. Yalin, X. Wu, M. Ashlie, Nanotechnology, 24 (2013) 375701.

[48] O. Hod, ChemPhysChem, 14 (2013) 2376-2391.

[49] H. Washizu, S. Kajita, M. Tohyama, T. Ohmori, N. Nishino, H. Teranishi, A. Suzuki, Faraday Discuss., 156 (2012) 279-291.

[50] X. Feng, S. Kwon, J.Y. Park, M. Salmeron, ACS Nano, 7 (2013) 1718-1724.

[51] K. Kikuchi, M. Yoshida, T. Maekawa, H. Watanabe, Chem. Phys. Lett., 185 (1991) 335-338.

[52] D. Berman, A. Erdemir, A.V. Sumant, Materials Today, 17 (2014) 31-42.

[53] O. Penkov, H.-J. Kim, H.-J. Kim, D.-E. Kim, Int. J. Prec. Eng. Manuf., 15 (2014) 577-585.

[54] W. Guo, J. Yin, H. Qiu, Y. Guo, H. Wu, M. Xue, Friction, 2 (2014) 209-225.

[55] J.C. Spear, J.P. Custer, J.D. Batteas, Nanoscale, (2015) in press. DOI:10.1039/C5NR01478F.

[56] P. Yitian, W. Zhuogiong, L. Cong, Nanotechnology, 25 (2014) 305701.

[57] G. Paolicelli, M. Tripathi, V. Corradini, A. Candini, S. Valeri, Nanotechnology, 26 (2015) 055703.

[58] B.J. Robinson, N.D. Kay, O.V. Kolosov, Langmuir, 29 (2013) 7735-7742.

[59] R. Muñoz, C. Gómez-Aleixandre, Chem. Vap. Deposition, 19 (2013) 297-322.

[60] Y. Zhang, L. Zhang, C. Zhou, Acc. Chem. Res., 46 (2013) 2329-2339.

[61] P. Egberts, G.H. Han, X.Z. Liu, A.T.C. Johnson, R.W. Carpick, ACS Nano, 8 (2014) 50105021.

[62] J. Yan, L. Yang, L. Bing, Y. Xiaofei, H. Tongwei, W. Ze, Nanotechnology, 23 (2012) 495703. [63] D. Berman, A. Erdemir, A.V. Sumant, Carbon, 59 (2013) 167-175. 
[64] D. Berman, S.A. Deshmukh, S.K.R.S. Sankaranarayanan, A. Erdemir, A.V. Sumant, Adv. Funct. Mater., 24 (2014) 6640-6646.

[65] H. Kinoshita, Y. Nishina, A.A. Alias, M. Fujii, Carbon, 66 (2014) 720-723.

[66] Y. Wang, J. Pu, L. Xia, J. Ding, N. Yuan, Y. Zhu, G. Cheng, Tribol. Lett., 53 (2014) 207-214.

[67] J. Pu, Y. Mo, S. Wan, L. Wang, Chem. Commun., 50 (2014) 469-471.

[68] T. Huang, Y. Xin, T. Li, S. Nutt, C. Su, H. Chen, P. Liu, Z. Lai, ACS Appl. Mater. Interfaces, 5 (2013) 4878-4891.

[69] L. Xuemei, Y. Jun, Z. Jianxin, G. Wanlin, Nanotechnology, 25 (2014) 105701.

[70] S. Chen, L. Brown, M. Levendorf, W. Cai, S.-Y. Ju, J. Edgeworth, X. Li, C.W. Magnuson, A. Velamakanni, R.D. Piner, J. Kang, J. Park, R.S. Ruoff, ACS Nano, 5 (2011) 1321-1327.

[71] L.H. Li, J. Cervenka, K. Watanabe, T. Taniguchi, Y. Chen, ACS Nano, 8 (2014) 1457-1462. [72] O. Tevet, P. Von-Huth, R. Popovitz-Biro, R. Rosentsveig, H.D. Wagner, R. Tenne, Proc. Natl. Acad. Sci. U.S.A., 108 (2011) 19901-19906.

[73] I. Lahouij, B. Vacher, F. Dassenoy, Lubr. Sci., 26 (2014) 163-173.

[74] I. Lahouij, B. Vacher, J.-M. Martin, F. Dassenoy, Wear, 296 (2012) 558-567.

[75] M. Ratoi, V. Niste, J. Walker, J. Zekonyte, Tribol. Lett., 52 (2013) 81-91.

[76] Z. Xu, K. Hu, C. Han, X. Hu, Y. Xu, Tribol. Lett., 49 (2013) 513-524.

[77] L. Yadgarov, V. Petrone, R. Rosentsveig, Y. Feldman, R. Tenne, A. Senatore, Wear, 297 (2013) 1103-1110.

[78] J. Kogovšek, M. Kalin, Tribol. Lett., 53 (2014) 585-597.

[79] X. He, H. Xiao, H. Choi, A. Díaz, B. Mosby, A. Clearfield, H. Liang, Colloids Surf., A, 452 (2014) 32-38.

[80] X. He, Y. Chen, H. Zhao, H. Sun, X. Lu, H. Liang, Friction, 1 (2013) 327-332. 


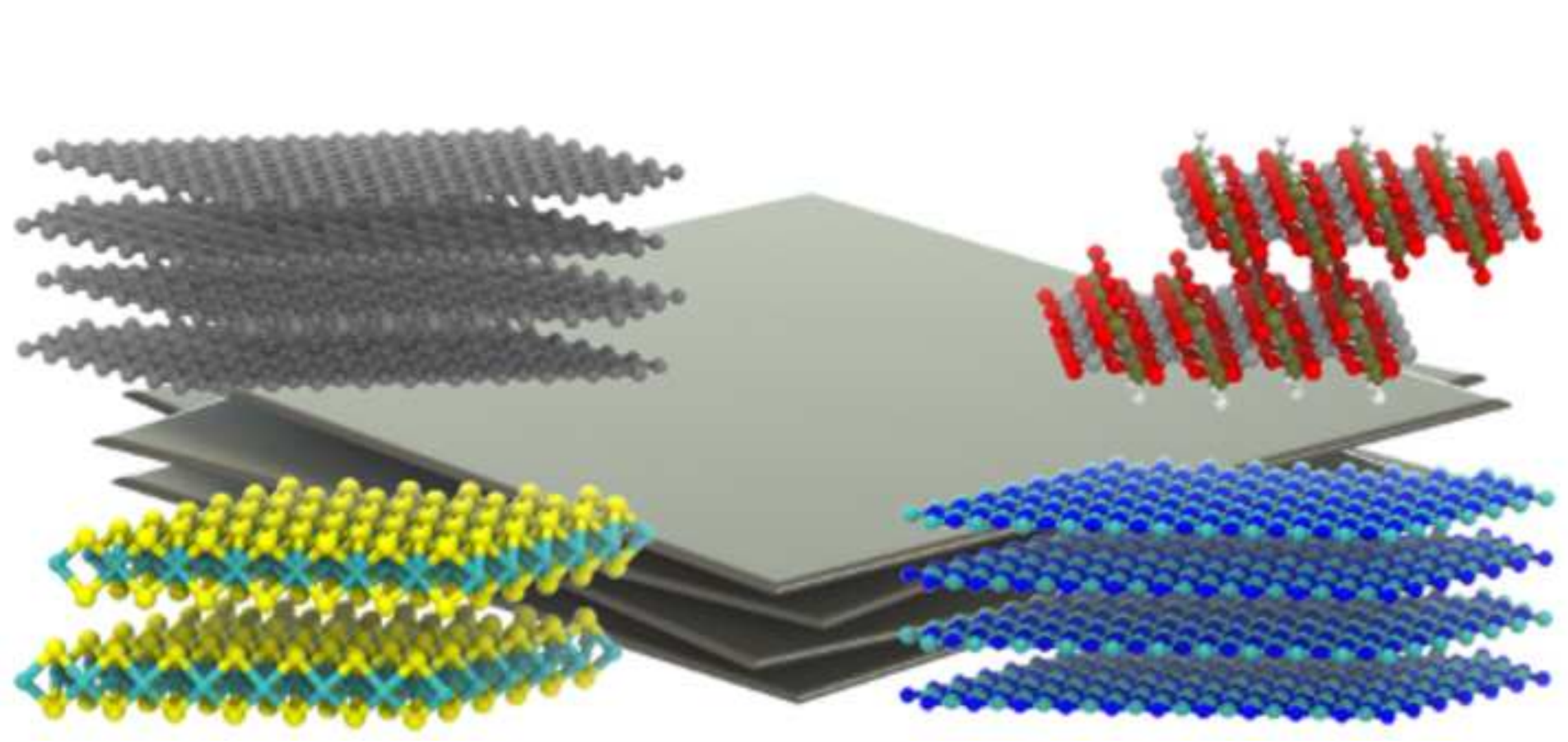

\section{Graphical Abstract (for review)}
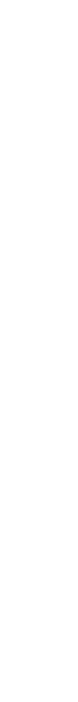